\title{
Happy with a difference, unhappy with an identity: Observers' mood determines processing depth in visual search
}

\author{
Anna Grubert • Petra Schmid • Joseph Krummenacher
}

Published online: 19 October 2012

(C) Psychonomic Society, Inc. 2012

\begin{abstract}
Visual search for feature targets was employed to investigate whether the mechanisms underlying visual selective attention are modulated by observers' mood. The effects of induced mood on overall mean reaction times and on changes and repetitions of target-defining features and dimensions across consecutive trials were measured. The results showed that reaction times were significantly slower in the negative than in the positive and neutral mood groups. Furthermore, the results demonstrated that the processing stage that is activated to select visual information in a feature search task is modulated by the observer's mood. In participants with positive or neutral moods, dimension-specific, but no feature-specific, intertrial transition effects were found, suggesting that these observers based their responses on a salience signal coding the most conspicuous display location. Conversely, intertrial effects in observers in a negative mood were feature-specific in nature, suggesting that these participants accessed the feature identity level before responding.
\end{abstract}

Keywords Selective attention · Visual search $\cdot$ Mood induction $\cdot$ Positive/negative mood $\cdot$ Salience $\cdot$ Object identity

The ability to select the information conducive to current behavioral goals and intentions from the abundance of

\footnotetext{
A. Grubert $\cdot$ J. Krummenacher $(\bowtie)$

Department of Psychology, University of Fribourg,

Rue de Faucigny 2,

1700 Fribourg, Switzerland

e-mail: joseph.krummenacher@unifr.ch

P. Schmid

Department of Psychology, University of Neuchâtel,

Neuchâtel, Switzerland
}

sensory stimuli provided by the environment is a key human cognitive skill. Effects of mood on visual information selection have been demonstrated in a series of studies in which mainly tasks investigating space- and object-based mechanisms of attentional selection have been employed. In the present study, employing a visual search task, the effects of mood on the processing mechanisms underlying feature-based selection were examined. The basic question was whether previously described mood-induced differences in selection processes would be mirrored on the very basal level of dimension- and feature-specific information selection. A specific technique (intertrial analysis) was applied in order to detect potential differences in the levels of processing engaged by observers in positive, negative, and neutral moods.

In a number of studies, mood-induced attentional biases were investigated by means of global-local paradigms such as Navon's (1977) letter task, in which global or local characteristics of large numerals composed of distinct small numerals had to be categorized. As an example, Gasper and Clore (2002, Exp.2) used Kimchi-Palmer figures (Kimchi \& Palmer, 1982), a variant of the Navon letter task. On each trial, observers were shown geometric objects such as triangles or squares that were composed of smaller triangles or squares (e.g., four triangles making the shape of a square). Then, participants were presented with a selection of two objects, one of which corresponded to the shape of the local elements of the object shown before (e.g., a triangle), while the other object corresponded to its global shape (e.g., a square). The task was to decide which of the two objects looked similar to the object that was presented initially. The results were straightforward: Participants in a negative mood, as compared to observers in positive or neutral moods, less often matched objects on the basis of the global characteristic. Gasper and Clore's finding is one example of a general pattern that indicates that mood systematically 
affects visual information selection, suggesting that a person in a positive mood tends to process global aspects of visual stimuli, while a person in a negative mood tends to process local aspects of visual information (e.g., Basso, Schefft, Ris, \& Dember, 1996; Derryberry \& Reed, 1998; Gasper \& Clore; 2002). In keeping with the global-local processing divide, it has been shown that the scope of focal attention is modulated by a person's mood: Negative mood narrows the focus of attention, whereas positive mood results in a broadened attentional focus (Christianson \& Loftus, 1990; Derryberry \& Reed, 1998; Derryberry \& Tucker, 1994; Easterbrook, 1959; Fredrickson, 2004; Rowe, Hirsh, \& Anderson, 2007; for a comprehensive review, see Förster \& Dannenberg, 2010). As an example, Rowe et al. tested the effect of mood on the scope of focal attention with the use of Eriksen and Eriksen's (1974) flanker task. After mood induction, participants were asked to identify, as quickly and accurately as possible, a target letter that was presented at the screen center and flanked by distractor letters at either side. The target and flanker letters were selected from two sets, each of which was associated with a predefined response. Flanker letters were either compatible (i.e., they required the same response as the response-relevant central letter) or incompatible with the response indicated by the central letter. Eriksen and Eriksen showed that reaction times (RTs) were significantly slower in incompatible than in compatible trials; moreover, the incompatibility effect was particularly strong when the flanker letters were presented at a distance close to the target letter. Rowe et al. found that, in the short target-flanker distance condition, the distractor incompatibility effect was significantly larger in participants in the positive as compared to the negative and neutral mood conditions. Importantly, if the target-flanker distance was increased, RTs in the positive mood group remained high, whereas the incompatibility effect was reliably reduced in the groups with neutral and negative moods. Rowe et al. concluded that with a large focus of attention, the distracting information from incompatible flanker letters was within the spatial extent of focal attention, thus causing interference with the relevant information at the level of response selection in the positive mood condition. In contrast, with narrow spatial attention, the flanker letters appeared outside the focus of attention; that is, they were deselected, and thus did not cause interference in observers with negative mood.

In line with the findings described above, it was also argued that the increased conceptual scope found in positive, relative to negative or neutral, affect leads to greater cognitive flexibility in a variety of tasks (for an overview, see Ashby, Isen, \& Turken, 1999). For example, Van der Stigchel, Imants, and Ridderinkhof (2011) showed increased stimulus inhibition in participants in a positive relative to those in a neutral mood in an antisaccade task (requiring the inhibition of a saccade to the target location and execution of an eye movement in the opposite direction). This result can be interpreted as enhanced top-down control, in terms of more successful suppression of irrelevant information in positive than in negative or neutral mood conditions. Ashby and colleagues (Ashby et al., 1999; Ashby, Valentin, \& Turken, 2002) proposed a neuropsychological theory to account for the improved cognitive control shown by observers in a positive mood. The authors assume that during periods of positive affect, the release of dopamine (mainly in the mesocorticolimbic system) is temporarily increased, and that raised dopamine levels in the prefrontal cortex entail greater cognitive flexibility. However, it was also found that the increase in cognitive flexibility comes at the cost of increased distractibility, which is reflected in, for example, higher error rates in participants with positive than with neutral or negative moods (Dreisbach, 2006; Dreisbach \& Goschke, 2004).

More recently, it has been shown that the relationship between an observer's affective state and the scope of the attentional focus is more variable than was originally assumed. As an example, Huntsinger (in press) found evidence for a broadened attentional spotlight in positive as compared to negative mood (i.e., increased flanker incompatibility effects) only if a global focus of attention was induced before the flanker task (by having observers report only the global numerals of Navon, 1977, stimuli). If, however, before the flanker task, participants were perceptually primed to adopt a local focus (by having them report only the local numerals of Navon stimuli), the flanker incompatibility effects were decreased in the positive as compared to the negative mood condition, reflecting a narrowed attentional scope in the positive relative to a broadened attentional focus in the negative mood condition. Furthermore, if no scope of the attention focus was made dominant (i.e., reporting equal proportions of global and local numerals in Navon stimuli), affect failed to modulate the spotlight of attention-there were equally strong flanker incompatibility effects in positive and negative moods. Huntsinger (in press; see also Huntsinger, Clore, \& Bar-Anan, 2010) concluded that there is no dedicated link between affect and the scope of the attentional focus. Rather, positive and negative moods lead to opposed effects on the way observers evaluate and validate their current thinking: Positive mood serves as a go signal, encouraging the use of the current mental content to focus on global or local aspects when, respectively, a global or local focus is relevant, therefore broadening or narrowing the spotlight of attention accordingly. Conversely, negative mood serves as a stop signal, discouraging the use of the current mental content, so that observers focus on 
local details when a global focus is dominant, and on global information when a local focus is dominant; therefore, the attentional focus is narrowed or broadened in contrast to the actual mental content. Huntsinger (in press; see also Clore \& Huntsinger, 2007) explained the deviation of their results from earlier findings by assuming that in a majority of the tasks used in the earlier studies, a general tendency to adopt a global focus was reinforced. This implies that positive mood was always found to be associated with global information processing (strengthening the current mental content afforded by a global focus), whereas negative mood was found to be associated with local information processing solely (counteracting the mental content conveyed by a global focus). Huntsinger et al.'s account is supported by a series of studies showing that, although observers are well able to switch between global and local processing modes, in the default case, a global attention focus is adopted (e.g., Kimchi, 1992; Kimchi \& Palmer, 1982; Navon, 1977; Navon \& Norman, 1983). In other words, according to Huntsinger and colleagues, the fixed effects of particular affective states on the attentional focus in earlier studies were due to the fact that the affective states had merged with the default mode of global attention.

Furthermore, Gable and Harmon-Jones (2008, 2010) showed that following the induction of positive mood in participants with high approach motivation (i.e., presenting observers with food pictures and inducing expectancies to receive the pictured food after the experiment), the attentional scope was narrower than in participants with low approach motivation (i.e., mere presentation of food items without inducing expectancies to actually receive this food). That is, the scope of attention following mood induction was modulated by an observer's motivational state. The concept of motivational intensity bears a relationship to differences in the levels of arousal associated with particular affective states. Jefferies, Smilek, Eich, and Enns (2008) showed that, in particular, the effects of negative mood critically depend on the level of arousal. In an attentional blink task (identification of letters presented in a stream of digits in rapid succession), accuracy was highest in participants in a sad mood (negative affective state, low arousal), lowest in anxious participants (negative affective state, high arousal), and intermediate in calm and happy observers (i.e., positive affective states and low or, respectively, high arousal). In the present experiment, we did not aim to introduce arousal as a dimension detached from affect, and therefore induced a happy mood as the positive affective state (with high arousal) and sad mood as the negative affective state (with low arousal). Induction of affective states-following the recommendation of the Rottenberg, Ray, and Gross (2007) film ratings catalogue-was achieved with the use of film clips that scored particularly high on the target emotions of either happiness or sadness, and particularly low on other emotions, such as anger, fear, or surprise. In the present experiment, potentially distinct effects of affect and arousal on levels of processing were conflated (positive affect with high arousal and negative affect with low arousal), and therefore need not be discussed separately.

\section{Dimension- and feature-based attentional selection}

The studies discussed above aimed at investigating mood-induced modulations of space-based (narrow vs. broad attentional spotlight: Huntsinger, in press; Rowe et al., 2007) or object-based (global vs. local processing styles: Basso et al., 1996; Derryberry \& Reed, 1998; Gable \& Harmon-Jones, 2008, 2010; Gasper \& Clore, 2002) mechanisms of attentional selection. However, influential theories of visual information selection have proposed that attentional selection can also be controlled by visual features such as color, orientation, size, and so forth (Huang \& Pashler, 2007; Treisman, 1988; Treisman $\&$ Gelade, 1980), or by dimension-based salience activations generated from visual features (Fecteau \& Munoz, 2006; Wolfe, 1994, 2007; Wolfe, Cave, \& Franzel, 1989). Investigations into the mechanisms underlying feature-based attention commonly employ visual search tasks in which observers are presented with arrays of objects (nontargets) in which a target object may or may not be embedded; participants are instructed to indicate, as quickly and accurately as possible, whether a target object is present or absent. In feature search tasks, the target differs from the nontargets by one salient feature, such as its color or orientation (e.g., a red vertical or green tilted bar among green vertical bars). Targets that may differ from distractors on one of several different dimensions are referred to as feature singletons. Wolfe (1994; Wolfe et al., 1989), in his influential guided search (GS) model, proposed that the allocation of focal attention (i.e., the detection of the target in feature search tasks) is controlled by a socalled salience representation (Crick, 1984; Koch \& Ullman, 1985). The information contained in the visual field is used to derive topographically organized representations of feature contrast or salience signals. These representations code how dissimilar or similar, with respect to a limited set of feature dimensions (e.g., color, orientation, etc.; see Wolfe \& Horowitz, 2004), a location in the visual field is relative to neighboring locations. For example, a red vertical bar presented 
among green vertical bars produces, at the location of the red bar, high feature contrast activation in the color dimension, but no feature contrast activation in the orientation dimension. Feature contrast signals are initially coded in independent dimension-specific representations or maps before being integrated into a supradimensional overall representation of salience. In other words, the more dissimilar that a location is compared to its neighboring locations, the higher is the integrated salience representation for that location (e.g., Krummenacher, Müller, \& Heller, 2001, 2002). Focal attention is directed to the most salient location first, then to the next most salient location, and so forth. The information at the attended location is gated to higher-level processes of object recognition and response generation. In feature search tasks, the only peak in the salience activation is at the location of the target, and focal attention is thus immediately directed to that location. Phenomenally, the target seems to "pop out" of the display.

However, it has been shown that search RTs on a current trial are affected by the target properties of the preceding trial (referred to as the intertrial or priming of pop-out effect; Maljkovic \& Nakayama, 1994, 1996). More specifically, Found and Müller (1996) found that search RTs for a red vertical target among green vertical objects on trial $n$ were expedited when the target on the preceding trial $n-1$ was also red and vertical, as compared to when the target was green and tilted. Furthermore, they showed that only the target-defining dimension (e.g., color or orientation), but not the targetdefining feature (e.g., red or blue, left- or right-tilted) affects performance across consecutive trials. Stated differently, changing the target dimension across trials (e.g., orientation on trial $n-1$, color on trial $n$ ) incurred an RT cost relative to repeating the dimension (e.g., color on $n-1$, color on $n$ ). By contrast, changing the target feature across trials (e.g., blue on $n-1$, red on $n$ ) did not incur any RT cost as compared to repeating the feature (e.g., red on $n-1$, red on $n$ ). The results suggest that in singleton feature search, responses are based on dimension-based salience signals; the exact featural identity of the target is not required for solving the task (i.e., to decide on the presence of absence of a feature target).

Found and Müller (1996) interpreted their finding in terms of Müller and colleagues' (Müller, Heller, \& Ziegler, 1995; Müller, Reimann, \& Krummenacher, 2003) dimension-weighting (DW) account of visual search. As in GS (Wolfe, 1994, 2007), dimension-based salience signals indicate the presence of a target, but in contrast to GS, DW assumes that the dimension-based salience signals need to be weighted by allocating a limited-capacity resource (attentional weight) to dimension-based processing modules. An increase in the weight in a given dimensional module (e.g., color) entails a reduction of the weights in other modules (e.g., orientation). Importantly, the distribution of attentional weights persists across trials. Therefore, if the target in two consecutive trials is defined on the same dimension, no time-consuming shift of weight is required, and RTs are thus faster than in sequences of trials that require a weight shift following a change of the target-defining dimension. As attentional weight operates at the level of dimension-based modules, a change of the target feature within the dimension does not affect the search RTs in detection tasks.

Extending Found and Müller's (1996) work, Krummenacher, Grubert, and Müller (2010) recently compared intertrial effects in conditions requiring feature detection or feature identification. In both conditions, participants had to decide whether or not a target item was present in the search display. In the feature detection task, they were presented with search displays consisting of an array of nontarget items (green vertical bars); on half of the trials, one of the nontargets was replaced by a feature target (a red or blue vertical or a green left-tilted or right-tilted bar). In the feature identification task, participants were presented with an isolated item (i.e., there was no context of nontarget items), which was defined by either a target (red, blue, left-tilted, or righttilted) or a nontarget (green vertical) feature. The results of the detection condition mirrored earlier findings of intertrial effects (Müller et al., 1995; Müller et al., 2003) and revealed dimension-based but no feature-based intertrial effects. Krummenacher et al. (2010) concluded that in the detection task, the target was discerned on the basis of the presence of a dimension-based salience signal; knowledge of the actual feature identity was not necessary to execute the correct response. By contrast, in the identification condition, feature-based intertrial effects were observed (in addition to dimension-based effects), indicating that participants needed to access the level of feature identities to decide whether the display item (shown in isolation) required a target or a nontarget response. Furthermore, the overall RTs were slower in the identification than in the detection condition. The authors reasoned that this RT cost was at least partially due to the fact that in the identification relative to the detection task, a perceptually finer-grained (and therefore more time-consuming) analysis of the single display item was required. To summarize, Krummenacher et al. (2010) showed that dimension- and feature-specific intertrial effects constitute a marker of the processing level-salience differences or feature identities-at which targets were processed. Processing at the level of dimension-based salience signals entails dimensionbased, but not feature-based, intertrial effects, whereas processing at the feature level is reflected by featurespecific intertrial effects. 


\section{Rationale of the study}

The objective of the present study was to investigate the effects of mood on feature-based mechanisms of attentional information selection. Positive (happy), negative (sad), or neutral mood was induced in observers prior to their completion of a visual feature search task. We examined the overall RTs and intertrial effects on RTs. In object-based selection tasks (e.g., a Navon letter task, Navon, 1977, or a Kimchi-Palmer figure task, Kimchi \& Palmer, 1982; see, e.g., Basso et al., 1996; Derryberry \& Reed, 1998; Gasper \& Clore; 2002), it was found that observers in a negative mood select local aspects of objects - that is, visual information is processed at a fine-grained level involving visual details-whereas observers in positive and neutral moods select global aspects of the objects - in other words, visual information is processed at a more general level, ignoring details. Translating these results to the concept of feature-based selection suggests that observers in a negative mood process visual items in a detailed way, corresponding to the level of feature values (identities), whereas participants in positive and neutral moods process visual items in a more perfunctory way, corresponding to the level of dimension-based salience signals. If people in a negative mood indeed access the representations of features, their performance should benefit from repetitions, across consecutive trials, of the exact target-defining feature, and performance should be impaired when the target-defining feature changes across trials. RT benefits and costs would be reflected in feature-based intertrial effects (see Krummenacher et al., 2010). In marked contrast, participants in positive and neutral moods should show no feature-based, but only dimension-based, intertrial effects, as, according to the DW account (Müller et al., 1995; Müller et al., 2003), their target-present responses are based on the presence of a peak of salience activation in one of the dimensional modules. Furthermore, we expected the overall mean RTs to be equally fast for observers in positive and neutral moods, but slower for observers in negative as compared to positive or neutral mood. Accessing the level of feature identity representations is more time-consuming than deciding on target presence on the basis of a dimension-based salience signal (Found \& Müller, 1996; Krummenacher et al., 2010). Accessing feature identity representations would constitute an explanation for (at least part of) the overall RT increase in the negative relative to the positive and neutral mood conditions. The presumption that overall RTs and intertrial effects in the positive and neutral conditions would not differ relies on previous findings showing that the average neutral mood of most people is rather positive (Diener \& Diener, 1996; Isen, 1984) and that processing levels in positive and neutral moods are therefore frequently the same (Gasper \& Clore, 2002; Navon, 1977). Furthermore, the assumption is also in line with Ashby and colleagues' (Ashby et al., 1999; Ashby et al., 2002) dopamine account, which states that a moderate concentration of dopamine is present in people in neutral affective states and that dopamine concentration is only increased in positive affective states.

\section{Method}

\section{Participants}

A group of 60 observers participated in the study. Of these, 20 observers were randomly assigned to each of the positive, negative, and neutral mood conditions; in each condition, 16 of the observers were female, and the age ranges (with mean ages) were 19-29 (23.3)years in the positive, 19-29 (22.5)years in the negative, and 19-38 (24.8)years in the neutral condition. All participants reported normal or corrected-to-normal vision, including color vision. They were paid at a rate of 10 Swiss francs (approximately \$11) per hour or received course credits.

\section{Stimuli, apparatus, and task}

The display consisted of an array of search items presented in cells $\left(2.0^{\circ} \times 2.0^{\circ}\right.$ of visual angle $)$ in an imaginary grid of $7 \times 7$ rows and columns (see Fig. 1). Item positions were jittered by a maximum of $0.6^{\circ}$ of visual angle relative to the cell centers. The minimum (and maximum) distances measured between the centers of gravity of the display items were $1.0^{\circ}\left(2.0^{\circ}\right)$ horizontally and vertically. The nontarget items were green vertical bars, and the target items differed from nontargets by either color (red or blue vertical bar) or orientation (green bar tilted $45^{\circ}$ to the left or right, relative to the vertical). Figure 1 shows example displays of the four possible target-present conditions; in target-absent trials (not shown), all of the display items were green vertical (nontarget) bars. On each trial, the target location was randomly chosen from among the inner $5 \times 5$ matrix cells, in order to keep the target-distractor feature contrasts comparable for all target locations (observers were not informed of this restriction). Each search item subtended $0.9^{\circ}$ of visual angle in height and $0.2^{\circ}$ in width, and the item colors were equiluminant $\left(1.6 \mathrm{~cd} / \mathrm{m}^{2}\right)$ green (CIE $x, y$ chromatic coordinates $0.311,0.578$ ), red (CIE $0.596,0.358$ ), and blue (CIE 0.148, 0.065). The background was black.

Stimulus presentation, timing, and response recording were controlled by a Pentium PC running the Windows 
Fig. 1 Schematic illustration of the different search displays used in the present study (top row, displays containing a color singleton target; bottom row, displays containing an orientation singleton target). The nontargets and left- and right-tilted targets were originally colored in green, and the color target features were red and blue
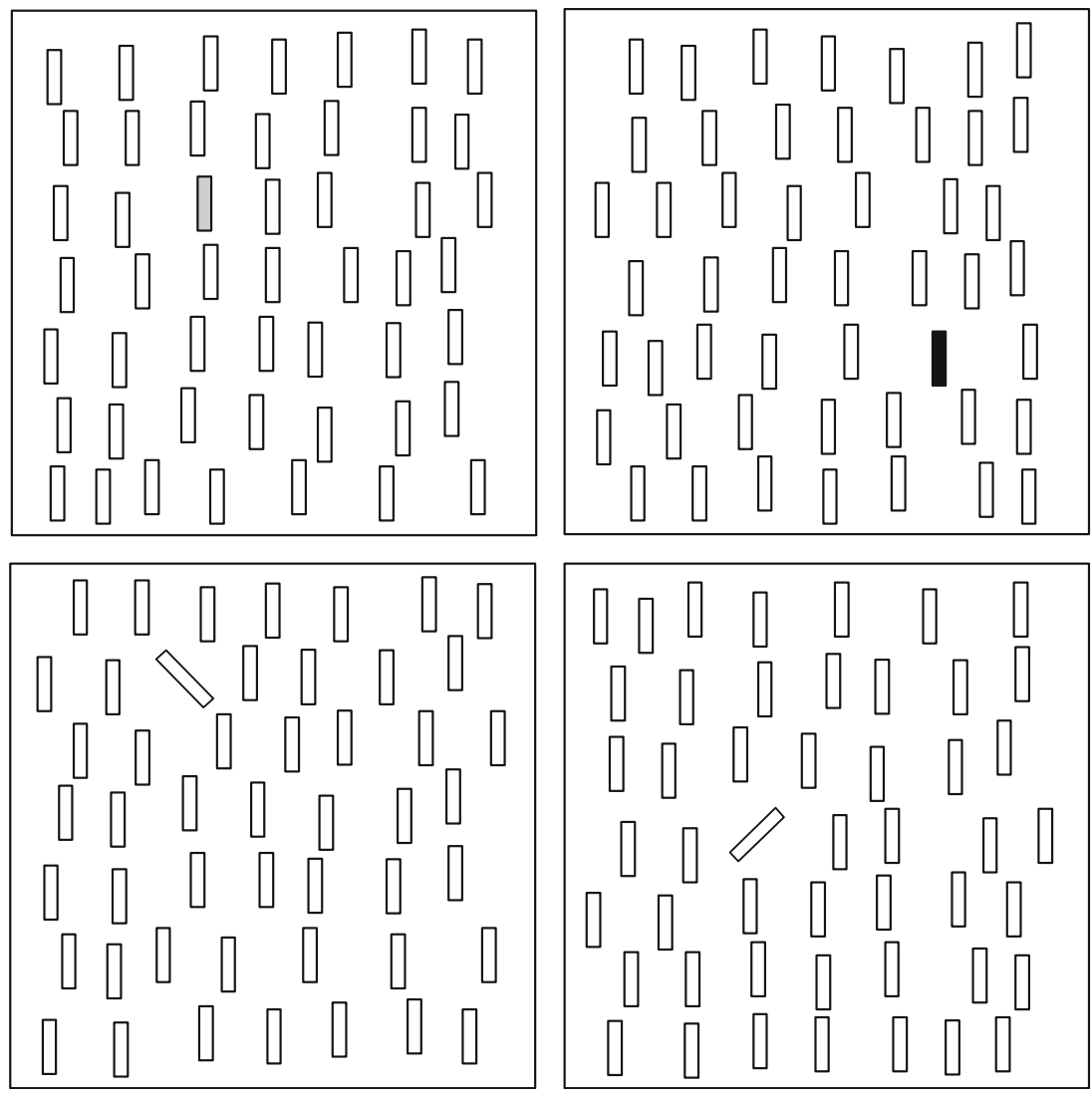

XP operating system and using the "Cogent 2000" toolbox (www.vislab.ucl.ac.uk/cogent.php) for MATLAB (The MathWorks Inc., Sherborn, MA). Displays were presented on a 19-in. CRT monitor (Phillips Brilliance P202) with a $100-\mathrm{Hz}$ screen refresh rate and a screen resolution of 1,280 $\times 1,024$ pixels. Observers viewed the display from a distance of about $70 \mathrm{~cm}$.

The observers' task was to indicate, as quickly and accurately as possible, whether or not a target item was present in the display. Target-present and target-absent responses were given manually, by pressing predefined keys on a standard keyboard.

The experiment comprised a total of 564 trials, divided into six blocks of 94 trials. In approximately $60 \%$ (or 336) of all trials, one of the nontarget items was replaced by a (color or orientation) feature singleton target; the remaining $40 \%$ (or 228) of the trials were target-absent trials. In each block, 48 (of the 56) targetpresent trials were presented in such a way that a minimum of eight (predefined) same-dimension, samefeature; eight same-dimension, different-feature; and eight different-dimension intertrial transition sequences occurred. The sequence of presentation of the targetpresent and target-absent trials was chosen randomly in each block. The four target types (red, blue, left-tilted, and right-tilted) were presented equally often.
Mood was induced using films. Participants watched a 3min film clip with a particular affective valence; additionally, they were instructed to adopt the mood state experienced by the film's protagonist. The films were chosen according to the Rottenberg et al. (2007) film-rating catalogue: When Harry Met Sally (Reiner, Scheinman, Stolt, \& Nicolaides, 1989) was used to induce positive (happy) mood, Return to Me (Tugend \& Hunt, 2000) to induce negative (sad) mood, and Sticks (a screensaver available for download at http://uweb.cas.usf.edu/mood/ images/sticks.mov) to induce neutral mood. The neutral condition was run as a baseline control.

\section{Procedure}

Prior to the visual search experiment proper, participants completed a block of 50 practice trials to become familiar with the visual search task. After the familiarization block, the observers watched a mood-inducing film clip and then completed the visual search experiment. After watching the clip, each observer rated it with respect to its affective valence $(1=$ very sad clip, $2=$ sad clip, $3=$ neutral clip, $4=$ happy clip , and $5=$ very happy clip). Furthermore, participants rated their current mood following completion of the familiarization block (initial mood) and after mood induction on a purpose-made five-item 
questionnaire $(1=$ very sad, $2=\operatorname{sad}, 3=$ neutral, $4=$ happy, and $5=$ very happy).

Each (familiarization and experimental) trial started with the presentation, for $500 \mathrm{~ms}$, of a fixation point (a circle with a diameter of $0.2^{\circ}$ of visual angle). Fixation was followed, for $200 \mathrm{~ms}$, by a blank screen and the onset of the search display, which remained visible until the observer's response (but for no longer than $3,000 \mathrm{~ms}$ ). Each response was followed by a blank-screen intertrial interval of $500 \mathrm{~ms}$ and by the next experimental trial.

\section{Results}

Exceedingly fast (below $200 \mathrm{~ms}$ ) and slow (above $1,600 \mathrm{~ms}$ ) RTs were excluded from the analysis (less than $1 \%$ of all trials); error trials were also excluded from the RT analysis. Data were analyzed using univariate and repeated measures analyses of variance (ANOVAs) and two-tailed $t$-tests; Bonferroni corrections were applied where necessary.

\section{Mood induction}

The material check showed that the mean ratings of the positive, negative, and neutral films scaled at 4.5 ([very] happy), 1.4 ([very] sad), and 3.0 (neutral), respectively. The mood induction was successful in that all of the individual ratings were in the expected directions. The mean ratings of initial and induced moods increased significantly $[t(19)=$ 2.6, $p=.033$ ] in the positive mood condition, from 3.7 (initial mood) to 4.1 (induced mood); decreased significantly $[t(19)=4.5, p<.001]$ in the negative mood condition, from 3.4 to 2.4 ; and did not differ $[3.3$ and $3.2 ; t(19)<1]$ in the neutral control condition.

\section{Reaction time intertrial effects}

Reaction time intertrial analyses were conducted to identify the levels (dimension-based salience or feature identity; see Krummenacher et al., 2010) at which search items were processed in the three mood conditions and to examine the hypothesis that observers in a negative mood process targets at the feature level, while observers in a positive mood base their responses on the presence of salience signals.

Three types of transitions across consecutive trials were examined: repetition of both the target dimension and feature (referred to as same dimension, same feature: $\mathrm{sDsF}$ ), repetition of the target dimension and change of the target feature (same dimension, different feature: $\mathrm{sDdF}$ ), and change of the target dimension (different dimension: $\mathrm{dD}$ ).
Intertrial effects on RTs were analyzed in a mixed-measures ANOVA with the within-subjects factor Intertrial Transition (sDsF, sDdF, or dD) and the between-subjects factor Mood (positive, negative, or neutral). The ANOVA revealed the two main effects to be significant. The main effect of intertrial transition $[F(2,114)=150.5, p<.001]$ indicated reliable effects of the previous trial on the RT of the current trial in all three mood conditions. The main effect of mood $[F(2,57)=7.0, p=.002]$ arose due to differences in the overall RTs of the three conditions: RTs were slowest in the negative mood condition $(470.6 \mathrm{~ms})$, followed by RTs in the neutral $(415.0 \mathrm{~ms})$ and positive $(398.1 \mathrm{~ms})$ mood conditions. More importantly, the interaction between intertrial transition and mood was significant $[F(4,114)=3.5, p=.011]$, showing that the magnitude of the intertrial effects depended on the observer's mood. Table 1 shows the magnitudes of the RT costs associated with dimension and feature changes in the three mood conditions. As predicted, RTs in all mood conditions were significantly slower when the target dimension changed across consecutive trials relative to when it was repeated (i.e., a dimension-based intertrial effect arising from a comparison of dD vs. $\mathrm{sDdF}$ transitions; see Table 1). By contrast, RTs increased significantly in feature-change as compared to feature-repetition trials only in the negative mood condition (i.e., a feature-based intertrial effect based on a comparison of SDdF vs. SDsF transitions; see Table 1). ${ }^{1}$ Overall, the results can be taken as evidence that observers in a negative mood access the level of feature representations to decide on the presence or absence of the target (see Krummenacher et al., 2010) even though there is no need to do so, as is demonstrated by observers in a positive or a neutral mood. ${ }^{2}$

Theoretically it is possible, however, that feature-based intertrial effects in the negative mood condition emerged because of the fact that overall RTs were higher in the

\footnotetext{
${ }^{1}$ The magnitude of the feature-based intertrial effect was about $13 \mathrm{~ms}$; in absolute numbers, the effect seems moderately large, and its significance was called into questioned by one of the reviewers. Note, however, that the effect is statistically significant $[t(19)=3.0, p=.016]$, and comparable in magnitude to the reliable feature-specific intertrial effects reported in Experiments 1 and 2 of Krummenacher et al. (2010).

${ }^{2}$ As the interpretation of the intertrial data is partially based on the absence of a reliable feature-based intertrial effect in the positive (nonsignificant difference of $5.1 \mathrm{~ms}$ ) and neutral (nonsignificant difference of $1.6 \mathrm{~ms}$ ) mood conditions, a power analysis was conducted to test the assumption that the power of the tests was sufficient to capture potentially significant feature-based effects in those two conditions (similar to the effect of $13.1 \mathrm{~ms}$ observed in the negative mood condition). The power of the test $(1-\beta)$ was calculated using the post-hoc option of the G*Power program (Faul, Erdfelder, Lang, \& Buchner, 2007). The analysis revealed an effect size $f$, as defined by Cohen (1988), of 0.71 , and a power of .92 for the negative mood condition. Thus, it can be concluded that the power of the test comparing the $\mathrm{sDdF}$ and $\mathrm{sDsF}$ conditions was sufficient to capture any effects of cross-trial feature changes in all mood conditions.
} 
Table 1 Intertrial effects on reaction times (RTs) for the positive, neutral, and negative mood conditions

\begin{tabular}{|c|c|c|c|c|c|c|c|c|c|}
\hline & \multicolumn{3}{|c|}{ Mean RTs (ms) } & \multicolumn{6}{|c|}{ Effect Size (ms) } \\
\hline & \multicolumn{3}{|c|}{ Intertrial Transition } & \multicolumn{3}{|c|}{ Dimension $(\mathrm{dD}-\mathrm{sDdF})$} & \multicolumn{3}{|c|}{ Feature $(\mathrm{sDdF}-\mathrm{sDsF})$} \\
\hline & $\mathrm{sDsF}$ & $\mathrm{sDdF}$ & $\mathrm{dD}$ & $\Delta \mathrm{ms}$ & $t(19)$ & $p$ & $\Delta \mathrm{ms}$ & $t(19)$ & $p$ \\
\hline Positive & 386.2 & 391.3 & 416.3 & 25.5 & 5.9 & $<.001$ & 5.1 & 2.0 & .123 \\
\hline Neutral & 405.6 & 404.0 & 435.5 & 31.5 & 10.1 & $<.001$ & 1.6 & 0.6 & 1.00 \\
\hline Negative & 451.0 & 464.1 & 496.8 & 32.7 & 7.6 & $<.001$ & 13.1 & 3.0 & .016 \\
\hline
\end{tabular}

Left-hand panel: RTs (in milliseconds) for the three intertrial transitions (sDsF, same dimension, same feature; sDdF, same dimension, different feature; $\mathrm{dD}$, different dimensions), separately for the three mood conditions (positive, neutral, and negative). Right-hand panel: Comparison of dimension change (dD) and feature change (sDdF) RT costs (including $t, d f$, and $p$ values)

negative than in the positive and neutral mood conditions. In order to rule out this possibility, the featurebased intertrial effects were compared separately for the faster versus the slower half of all RTs (based on mean splits of the individual RTs) in all of the three mood conditions. The results replicated the pattern of intertrial effects of the overall RT analysis: The ANOVAs for both the fast and the slow RTs revealed significant main effects of intertrial transition [fast RTs, $F(2,114)=$ 34.2; slow RTs, $F(2,114)=14.1$; both $p$ s $<.001]$ and of mood [fast RTs, $F(2,57)=4.8, p=.012$; slow RTs, $F$ $(2,57)=9.7, p<.001]$, as well as significant interactions [fast RTs, $F(4,114)=3.1, p=.019$; slow RTs, $F(4$, $114)=3.0, p=.022]$. Follow-up $t$-tests revealed statistically reliable dimension-based intertrial effects in all $\operatorname{mood}$ groups for fast [positive, $t(19)=2.9, p=.019$; neutral, $t(19)=2.8, p=.021$; negative, $t(19)=3.1$, $p=.013$ ] as well as slow [positive, $t(19)=3.0$, $p=.014$; neutral, $t(19)=2.4, p=.050$; negative, $t(19)=5.1, p<.001]$ RTs. Importantly, the featurebased intertrial effects were statistically reliable in the negative mood condition for fast $[t(19)=2.9$, $p=.019]$ as well as for slow $[t(19)=2.5, p=.048)$ RTs, while they were not statistically significant in the positive and neutral mood conditions for either fast [positive, $t(19)<1$; neutral, $t(19)=1.0, p=.643$ ] or slow [positive, $t(19)=1.9, p=.137$; neutral, $t(19)<1]$ RTs. On the basis of the present results, the interpretation can be ruled out that the contrast between statistically significant feature-based intertrial effects in the negative mood condition and the absence of featurebased effects in the positive and neutral mood conditions is an effect of general response speed. Under the assumption that the feature-based intertrial effects were due to slow RTs, the feature-based effects would not be expected to emerge for the fast RTs in the negative mood condition; rather, they would be expected to appear for the slow RTs in the positive and neutral mood conditions. In sum, the reliable feature-based intertrial effects observed in the negative mood condition are not due to, but can be taken to reflect a cause (the processing of feature identities) for the overall RT increase in the negative relative to the positive and neutral mood conditions.

\section{Reaction times}

The mean target-present and target-absent trial RTs in the positive, negative, and neutral mood conditions (see Fig. 2) were analyzed in a mixed-measures ANOVA with the within-subjects factor Trial (present or absent) and the between-subjects factor Mood (positive, negative, or neutral). The main effect of mood was statistically significant $[F(2,57)=9.7, p<.001]$. In detail, negative mood condition RTs $(539.7 \mathrm{~ms})$ were significantly slower than the positive [434.4 ms; $t(38)=3.8, p=.002]$ and neutral [462.7 ms; $t(38)=2.7, p=.032] \operatorname{mood}$ condition RTs, while the positive and neutral mood condition RTs were statistically not different $[t(38)=1.8, p=.233] .^{3}$

The main effect of trial $[F(1,57)=72.3, p<.001]$ and the Mood $\times$ Trial interaction $[F(2,57)=5.0, p=.010]$ were also statistically significant. Follow-up $t$-tests showed that in all mood conditions, RTs in target-absent trials were markedly slower than those in target-present trials (positive, $454.4 \mathrm{~ms}$ vs. $414.4 \mathrm{~ms}, t(19)=4.4$; neutral, $490.5 \mathrm{~ms}$ vs. $435.0 \mathrm{~ms}, t(19)=7.9$; negative, 587.8 vs. $491.5 \mathrm{~ms}, t(19)=4.9$; all $p \mathrm{~s}<.001$; see Fig. 2). The RT differences for target-present relative to target-absent trials

\footnotetext{
${ }^{3}$ In order to show that the RT increase in the negative mood condition was independent of the target dimension, an ANOVA with the factors Target Identity (color or orientation) and Mood (positive, neutral, or negative) was conducted. We found significant main effects of mood $[F(2,57)=7.9, p=.001]$ and target identity $[F(1,57)=164.4, p<.001]$, but no reliable interaction $[F(2,57)<1]$. In other words, RTs to color targets $(434.4 \mathrm{~ms})$ were faster than RTs to orientation targets $(459.5 \mathrm{~ms})$; however, the processing times were affected by the observers' mood independently of the target's dimensional definition.
} 


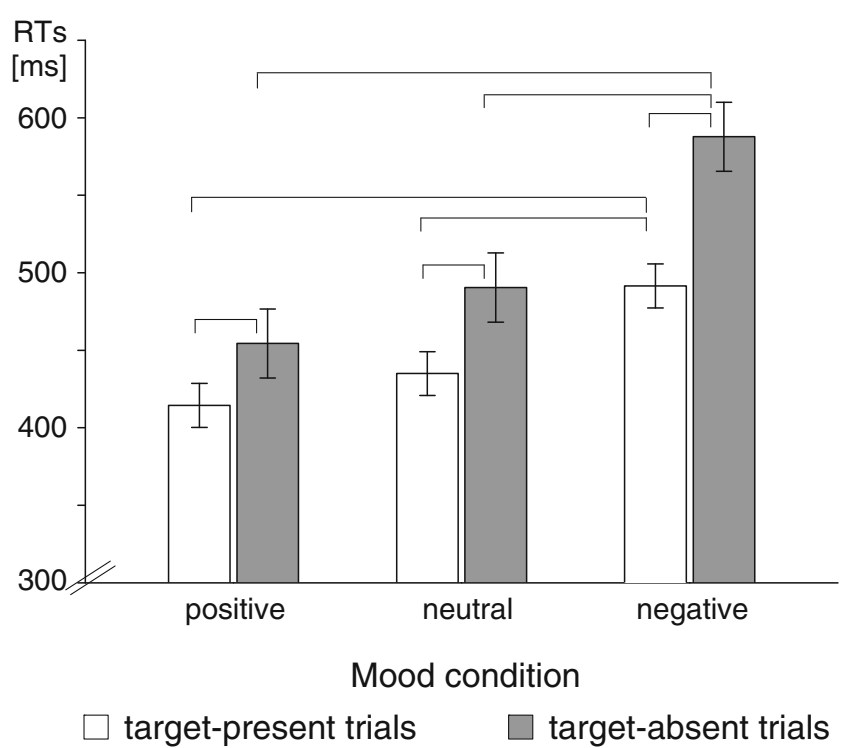

Fig. 2 Reaction times (RTs) and standard errors of the means (in milliseconds) for target-present and target-absent trials, presented separately for the positive, neutral, and negative mood conditions. The brackets indicate statistically significant $(p<.05)$ differences

were compared across mood conditions (positive, $40.0 \mathrm{~ms}$; neutral, $55.5 \mathrm{~ms}$; negative, $96.3 \mathrm{~ms}$ ) and were found to be larger in the negative than in the positive mood condition $[t(38)=2.6, p=.037]$, but statistically they were not different in the negative and neutral mood conditions $[t(38)=2.0, p=.168]$ and in the positive and neutral mood conditions $[t(38)=1.4, p=.544]$. (For an interpretation, refer to the "Discussion" section below.)

\section{Error rates}

A univariate ANOVA of the overall error rates with the between-subjects factor Mood (positive, negative, or neutral) did not reveal a significant main effect $[F(2$, $57)=1.6, p=.217]$, although error rates in the positive mood condition $(4.4 \%)$ were slightly higher than those in the negative $(3.1 \%)$ and neutral (3.4\%) mood conditions. The slight increase in error rates in the positive mood condition is in line with the assumptions of Dreisbach (2006; Dreisbach \& Goschke, 2004), who suggested that observers in a positive mood are more easily distracted, and therefore produce higher error rates, than observers in a negative or neutral mood. Errors in target-present (misses) and target-absent (false alarms) trials were analyzed in a mixed-measures ANOVA with the within-subjects factor Error Type (miss or false alarm) and the between-subjects factor Mood (positive, negative, or neutral). Here, the main effect of error type was statistically significant $[F(1,57)=20.4$, $p<.001]$ : In all three mood conditions, false alarm rates were higher than miss rates.

\section{Discussion}

In the present experiment, we demonstrated that the mechanisms of feature-based attentional selection are modulated by observers' moods. The mean search RTs of observers in a negative mood showed that processing was slowed dramatically, as compared to positive or neutral moods, while comparable RTs in the positive and neutral mood conditions suggested equally efficient processing. The processing differences between the negative and the positive and neutral mood conditions were examined by analyses of the intertrial transition effects. The intertrial analysis revealed that observers in positive and neutral moods, as predicted by previous studies (e.g., Found \& Müller, 1996; Krummenacher et al. 2010), showed dimension-based, but no feature-based, intertrial effects. By contrast, changing the target feature (within a dimension) incurred an RT cost in the negative mood condition only. Dimension-based change effects were expected in all conditions, as the presence of salience activity in the modules processing either color or orientation would need to be established by a mechanism requiring the time-consuming shift of attentional weight between dimensions in the case of a dimension change across trials (Müller et al., 1995; Müller et al., 2003). The presence of a salience activation signaling the presence of a singleton target in one of the two target-defining dimensions was sufficient to trigger a target-present response in the positive and neutral mood conditions. By contrast, RT costs associated with a feature change suggested that the target item was processed at the level of feature identity in participants with a negative mood (see Krummenacher et al., 2010). Rather than relying on the presence of salience activation for a speeded response, as did observers in a positive or neutral mood, participants in a negative mood accessed the target's featural value for a thorough analysis of its identity.

The present findings are in line with the results of previous experiments investigating the effects of affect on information selection (e.g., Basso et al., 1996; Derryberry \& Reed, 1998; Gasper \& Clore, 2002; Rowe et al., 2007), which showed that participants in positive (and neutral) moods employ a spatial spotlight with a wide scope and process global (low-resolution) aspects of visual stimuli, while participants in negative moods use an attentional spotlight with a narrow focus and process local (high-resolution) aspects of visual information. Note also that, although our results replicated the finding of a fixed pattern between (positive and negative) affect and (global and local) processing styles with dedicated (wide and narrow) foci of attention, they are also in keeping with the findings of 
Huntsinger (in press) and of Gable and Harmon-Jones $(2008,2010)$, who suggested that the links between these variables are flexible. Huntsinger found a wide or a narrow scope of attention, respectively, associated with positive or negative mood in conditions in which observers were made to adopt a global focus only. When observers adopted a local focus, or when neither a global nor a local focus was induced, this relationship pattern was reversed or abolished. Furthermore, Gable and Harmon-Jones $(2008,2010)$ showed that, under conditions of high approach motivation (i.e., with a desire to obtain an attractive reward), positive mood could also result in a narrow attentional focus. However, we did not specify a particular (global or local) focus in our experiment. This means that in our experimental context, the adoption of a general global focus was reinforced (e.g., Kimchi, 1992; Kimchi \& Palmer, 1982; Navon, 1977; Navon \& Norman, 1983), and therefore positive and negative affect in our experiment were conflated with the "usually dominant [global] way of viewing the world" (Huntsinger, in press, p.4). Also, we induced emotional states with low approach motivation by simply exposing observers to film clips with a positive, neutral, or negative emotional valence. Thus, we created exactly the conditions under which Huntsinger (in press) and Gable and Harmon-Jones $(2008,2010)$ predicted fixed patterns of narrowed and enlarged spotlights in participants with, respectively, negative and positive mood.

The pattern of RT intertrial effects provides evidence that the more time-consuming access of feature value representations in observers with negative relative to positive and neutral moods is the main source of the increase in the overall RTs of observers with a negative affective state. However, the significantly more pronounced difference between target-present and target-absent trial RTs in observers in a negative as compared to a positive or neutral mood constitutes another source of the overall RT increase. Slower search RTs in target-absent than in target-present trials have been reported repeatedly in feature search tasks. Krummenacher and colleagues (e.g., Krummenacher et al., 2010; Krummenacher et al., 2001, 2002) speculated that longer absent-trial RTs are due to observers' refraining from responding during a short additional interval at about the time that a (target-present) response is usually initiated. This delay could allow for the processing of salience activations with a low probability of signaling the target, and thus reduce the likelihood of missing a target. Chun and Wolfe (1996), in an analysis of the mechanism underlying targetabsent responses, argued that observers flexibly shift a threshold that differentiates salience signals with a given probability of indicating a target item from salience activations that are unlikely to signal a target. As an example, the threshold is lowered as an immediate response to a target miss error. Lowering the threshold results in a larger number of items being sampled as potential targets and is mirrored in an RT increase. The present results suggest that observers in a negative mood consistently use a lower "target candidate" threshold than do observers in a positive mood. A lower threshold for target candidate signals would be reflected by increased search RT variance in the negative as compared to the positive mood conditions. This prediction was tested in additional analyses of the RT standard deviations across mood conditions. Standard deviations in the negative mood condition $(119.4 \mathrm{~ms})$ differed significantly from those in the positive [73.9 ms; $t(38)=4.5, p<.001]$ and neutral [84.2 ms; $t(38)=3.5, p=.004]$ mood conditions. [The standard deviations of the positive and neutral mood conditions did not differ statistically: $t(38)=1.8, p=.230$.] With error rates being statistically the same in both positive and negative mood conditions, the RT difference suggests that the threshold adaptation in negative mood was required to achieve the instructed level of accuracy.

In previous work, participants in a negative mood have been described as having a narrower attentional focus than do observers in a positive mood (Christianson \& Loftus, 1990; Derryberry \& Reed, 1998; Derryberry \& Tucker, 1994; Easterbrook, 1959; Fredrickson, 2004; Rowe et al., 2007). A narrow focus of attention could be the consequence of participants' need to process the relevant information at the feature level in order to access the target's identity. Conversely, a narrow focus could also be the very reason why objects are processed thoroughly (i.e., at the feature level). Lavie (1995; Lavie \& Tsal, 1994) argued that cognitive load determines the amount of information that is processed. In low-load conditions, irrelevant distractor information is processed together with relevant target information, entailing interference. In connection with negative mood, a narrow focus of the attention spotlight might result in a highly efficient rejection of distractor information outside of the focus, and, as a consequence, result in the capacity to process relevant information at the level of feature representations.

In conclusion, the difference in overall RTs in feature search between participants in a positive, a neutral, or a negative mood can be explained by the level of information processing: Observers in a positive mood are happy to base their response on the presence of a salience difference, while participants in a negative mood base their responses on the identity of the target item.

Author Note A.G. is now at Birkbeck College, University of London. P.S. is now at New York University. The project was supported by Swiss National Science Foundation (SNSF) Grant Nos. PP001110543/1 (to J.K.) and PBFRP1-133492 (to A.G.). The authors thank Todd Horowitz and three anonymous reviewers for their insightful comments on an earlier version of the manuscript. 


\section{References}

Ashby, F. G., Isen, A. M., \& Turken, A. U. (1999). A neuropsychological theory of positive affect and its influence on cognition. Psychological Review, 106, 529-550. doi:10.1037/0033295X.106.3.529

Ashby, F. G., Valentin, V. V., \& Turken, A. U. (2002). The effects of positive affect and arousal on working memory and executive attention: Neurobiology and computational models. In S. Moore \& M. Oaksford (Eds.), Emotional cognition: From brain to behaviour (pp. 245-287). Amsterdam, The Netherlands: Benjamins.

Basso, M. R., Schefft, B. K., Ris, M. D., \& Dember, W. N. (1996). Mood and global-local visual processing. Journal of the International Neuropsychological Society, 2, 249-255. doi:10.1017/S1355617700001193

Christianson, S.-Å., \& Loftus, E. F. (1990). Some characteristics of people's traumatic memories. Bulletin of the Psychonomic Society, 28, 195-198.

Chun, M. M., \& Wolfe, J. M. (1996). Just say no: How are visual searches terminated when there is no target present? Cognitive Psychology, 30, 39-78. doi:10.1006/cogp.1996.0002

Clore, G. L., \& Huntsinger, J. R. (2007). How emotion informs judgments and regulates thought. Trends in Cognitive Sciences, 11, 393-399. doi:10.1016/j.tics.2007.08.005

Cohen, J. (1988). Statistical power analysis for the behavioral sciences (2nd ed.). Hillsdale, NJ: Erlbaum.

Crick, F. (1984). Function of the thalamic reticular complex: The searchlight hypothesis. Proceedings of the National Academy of Sciences, 81, 4586-4590.

Derryberry, D., \& Reed, M. A. (1998). Anxiety and attentional focusing: Trait, state and hemispheric influences. Personality and Individual Differences, 25, 745-761. doi:10.1016/S01918869(98)00117-2

Derryberry, D., \& Tucker, D. M. (1994). Motivating the focus of attention. In P. M. Niedenthal \& S. Kitayama (Eds.), The heart's eye: Emotional influences in perception and attention (pp. 167196). San Diego, CA: Academic Press.

Diener, E., \& Diener, C. (1996). Most people are happy. Psychological Science, 7, 181-185. doi:10.1111/j.1467-9280.1996.tb00354.x

Dreisbach, G. (2006). How positive affect modulates cognitive control: The costs and benefits of reduced maintenance capability. Brain and Cognition, 60, 11-19. doi:10.1016/j.bandc.2005.08.003

Dreisbach, G., \& Goschke, T. (2004). How positive affect modulates cognitive control: Reduced perseveration at the cost of increased distractibility. Journal of Experimental Psychology: Learning, Memory, and Cognition, 30, 343-353. doi:10.1037/02787393.30.2.343

Easterbrook, J. A. (1959). The effect of emotion on cue utilization and the organization of behavior. Psychological Review, 66, 183-201. doi: $10.1037 / \mathrm{h} 0047707$

Eriksen, B. A., \& Eriksen, C. W. (1974). Effects of noise letters upon the identification of a target letter in a nonsearch task. Attention, Perception, \& Psychophysics, 16, 143-149. doi:10.3758/ BF03203267

Faul, F., Erdfelder, E., Lang, A.-G., \& Buchner, A. (2007). G*Power 3: A flexible statistical power analysis program for the social, behavioral, and biomedical sciences. Behavior Research Methods, 39, 175-191. doi:10.3758/BF03193146

Fecteau, J. H., \& Munoz, D. P. (2006). Salience, relevance, and firing: A priority map for target selection. Trends in Cognitive Sciences, 10, 382-390. doi:10.1016/j.tics.2006.06.011

Förster, J., \& Dannenberg, L. (2010). GLOMOsys: A systems account of global versus local processing. Psychological Inquiry, 21, 175197. doi:10.1080/1047840X.2010.487849
Found, A., \& Müller, H. J. (1996). Searching for unknown feature targets on more than one dimension: Investigating a "dimensionweighting" account. Perception \& Psychophysics, 58, 88-101. doi:10.3758/BF03205479

Fredrickson, B. L. (2004). The broaden-and-build theory of positive emotions. Philosophical Transactions of the Royal Society B, 359, 1367-1377. doi:10.1098/rstb.2004.1512

Gable, P. A., \& Harmon-Jones, E. (2008). Approach-motivated positive affect reduces breadth of attention. Psychological Science, 19, 476-482. doi:10.1111/j.1467-9280.2008.02112.x

Gable, P. A., \& Harmon-Jones, E. (2010). The blues broaden, but the nasty narrows: Attentional consequences of negative affects low and high in motivational intensity. Psychological Science, 21, 211-215. doi:10.1177/0956797609359622

Gasper, K., \& Clore, G. L. (2002). Attending to the big picture: Mood and global versus local processing of visual information. Psychological Science, 13, 34-40. doi:10.1111/1467-9280.00406

Huang, L., \& Pashler, H. (2007). Working memory and the guidance of visual attention: Consonance-driven orienting. Psychonomic Bulletin \& Review, 14, 148-153. doi:10.3758/BF03194042

Huntsinger, J.R. (in press). Does positive affect broaden and negative affect narrow attentional scope? A new answer to an old question. Journal of Experimental Psychology: General. doi:10.1037/ a0027709

Huntsinger, J. R., Clore, G., \& Bar-Anan, Y. (2010). Mood and global local focus: Priming a local focus reverses the link between mood and global-local processing. Emotion, 10, 722-726. doi:10.1037/ a0019356

Isen, A. M. (1984). Toward understanding the role of affect in cognition. In R. Wyer \& T. Srull (Eds.), Handbook of social cognition (pp. 179-236). Hillsdale: Erlbaum.

Jefferies, L. N., Smilek, D., Eich, E., \& Enns, J. T. (2008). Emotional valence and arousal interact in attentional control. Psychological Science, 19, 290-295. doi:10.1111/j.1467-9280.2008.02082.x

Kimchi, R. (1992). Primacy of wholistic processing and global/local paradigm: A critical review. Psychological Bulletin, 112, 24-38. doi:10.1037/0033-2909.112.1.24

Kimchi, R., \& Palmer, S. E. (1982). Form and texture in hierarchically constructed patterns. Journal of Experimental Psychology: Human Perception and Performance, 8, 521535. doi:10.1037/0096-1523.8.4.521

Koch, C., \& Ullman, S. (1985). Shifts in selective visual attention: Towards the underlying neural circuitry. Human Neurobiology, 4, 219-227.

Krummenacher, J., Grubert, A., \& Müller, H. J. (2010). Intertrial and redundant-signals effects in visual search and discrimination tasks: Separable pre-attentive and post-selective effects. Vision Research, 50, 1382-1395. doi:10.1016/j.visres.2010.04.006

Krummenacher, J., Müller, H. J., \& Heller, D. (2001). Visual search for dimensionally redundant pop-out targets: Evidence for parallelcoactive processing of dimensions. Perception \& Psychophysics, 63, 907-917. doi:10.3758/BF03194446

Krummenacher, J., Müller, H. J., \& Heller, D. (2002). Visual search for dimensionally redundant pop-out targets: Parallel-coactive processing of dimensions is location-specific. Journal of Experimental Psychology: Human Perception and Performance, 28, 1303-1322. doi:10.1037/0096-1523.28.6.1303

Lavie, N. (1995). Perceptual load as a necessary condition for selective attention. Journal of Experimental Psychology: Human Perception and Performance, 21, 451-468. doi:10.1037/0096-1523.21.3.451

Lavie, N., \& Tsal, Y. (1994). Perceptual load as a major determinant of the locus of selection in visual attention. Perception \& Psychophysics, 56, 183-197. doi:10.3758/BF03213897

Maljkovic, V., \& Nakayama, K. (1994). Priming of pop-out: I. Role of features. Memory \& Cognition, 22, 657-672. doi:10.3758/ BF03209251 
Maljkovic, V., \& Nakayama, K. (1996). Priming of pop-out: II. The role of position. Perception \& Psychophysics, 58, 977-991. doi:10.3758/BF03209251

Müller, H. J., Heller, D., \& Ziegler, J. (1995). Visual search for singleton feature targets within and across feature dimensions. Perception \& Psychophysics, 57, 1-17. doi:10.3758/ BF03211845

Müller, H. J., Reimann, B., \& Krummenacher, J. (2003). Visual search for singleton feature targets across dimensions: Stimulus- and expectancy-driven effects in dimensional weighting. Journal of Experimental Psychology: Human Perception and Performance, 29, 1021-1035. doi:10.1037/ 0096-1523.29.5.1021

Navon, D. (1977). Forest before trees: The precedence of global features in visual perception. Cognitive Psychology, 9, 353-383. doi:10.1016/0010-0285(77)90012-3

Navon, D., \& Norman, J. (1983). Does global precedence reality depend on visual angle? Journal of Experimental Psychology: Human Perception and Performance, 9, 955-965. doi:10.1037/ 0096-1523.9.6.955

Reiner, R. (Producer/Director), Scheinman, A., Stolt, J., \& Nicolaides, S. (Producers) (1989). When Harry met Sally [Motion pictures]. Hollywood, CA: New Line Home Video.

Rottenberg, J., Ray, R. D., \& Gross, J. J. (2007). Emotion elicitation using films. In J. A. Coan \& J. J. B. Allen (Eds.), The handbook of emotion elicitation and assessment (pp. 9-28). New York: Oxford University Press.

Rowe, G., Hirsh, J. B., \& Anderson, A. K. (2007). Positive affect increases the breadth of attentional selection. Proceedings of the
National Academy of Sciences, 104, 383-388. doi:10.1073/ pnas.0605198104

Treisman, A. (1988). Features and objects: The fourteenth bartlett memorial lecture. Quarterly Journal of Experimental Psychology, 40, 201-237. doi:10.1080/02724988843000104

Treisman, A., \& Gelade, G. (1980). A feature-integration theory of attention. Cognitive Psychology, 12, 97-136. doi:10.1016/00100285(80)90005-5

Tugend, J. L. (Producer), \& Hunt, B. (Director) (2000). Return to me [Motion picture]. Hollywood, CA: Metro-Goldwyn-Mayer Pictures.

Van der Stigchel, S., Imants, P., \& Ridderinkhof, K. R. (2011). Positive affect increases cognitive control in the antisaccade task. Brain and Cognition, 75, 177-181. doi:10.1016/ j.bandc.2010.11.007

Wolfe, J. M. (1994). Guided Search 2.0: A revised model of visual search. Psychonomic Bulletin \& Review, 1, 202-238. doi:10.3758/ BF03200774

Wolfe, J. M. (2007). Guided Search 4.0: Current progress with a model of visual search. In W. Gray (Ed.), Integrated models of cognitive systems (pp. 99-119). Oxford University Press: New York.

Wolfe, J. M., Cave, K. R., \& Franzel, S. L. (1989). Guided search: An alternative to the feature integration model for visual search. Journal of Experimental Psychology: Human Perception and Performance, 15, 419-433. doi:10.1037/0096-1523.15.3.419

Wolfe, J. M., \& Horowitz, T. S. (2004). What attributes guide the deployment of visual attention and how do they do it? Nature Reviews Neuroscience, 5, 495-501. doi:10.1038/nrn1411 